\title{
A Report on Spring Migration in the Regina Area, 1958
}

by Fred W. Lahrman, Saskatchewan Museum of Natural History

Members of the Museum staff and of the Bird Group in the Regina Natural History Society co-operated his year in a special study of the ppring migration in the Regina area. Each individual reported his observations to the Museum where I kept $\mathrm{r}$ zeord of early arrival dates. This ist is particularly interesting because it represents the joint findings of a number of enthusiastic birders. The ist includes records submitted to April 30. This year special interest has been shown in the Regina area because Regina birders want to become more familiar with local conditions in view of the A.O.U. Meeting to be held here in August, 1959. We expect that this renewed interest in bur local area will stimulate "birding" activities throughout the summerpoth this year and next.

\section{Early Arrival Dates, Spring 1958}

\begin{tabular}{|c|c|}
\hline Date & Species \\
\hline April 18 & Horned Grebe (3) \\
\hline April 19 & Pied-billed Grebe (1) \\
\hline April 20 & White Pelican (flocks) \\
\hline April 21 & Double-crested Cormorant (1) \\
\hline April 2 & Great Blue Heron (1) \\
\hline April 25 & Common Egret (1) \\
\hline March 29 & Whistling Swan (5) \\
\hline March 30 & Canada Goose (32) \\
\hline April 6 & White-fronted Goose (flock) \\
\hline April 8 & Snow Goose $(75+)$ \\
\hline April 8 & Blue Goose $(75+)$ \\
\hline April 9 & Gadwall (2) \\
\hline March 25 & Pintail (12) \\
\hline April 1 & Green-winged Teal (1) \\
\hline April 18 & Blue-winged Teal (2) \\
\hline March 30 & American Widgeon (2) \\
\hline March 26 & Shoveler (4) \\
\hline April 8 & Redhead ( 5 ) \\
\hline pril 8 & Ring-necked Duck (1) \\
\hline pril 1 & Canvasback (5) \\
\hline April 1 & Lesser Scaup ( 8 ) \\
\hline March 25 & Common Goldeneye ( 3 ) \\
\hline April 21 & Bufflehead (1) \\
\hline April 25 & Ruddy Duck (3) \\
\hline March 31 & Common Merganser (4) \\
\hline April 5 & Red-breasted Merganser (1) \\
\hline April 22 & Turkey Vulture (1) \\
\hline April 19 & Cooper's Hawk (1) \\
\hline March 30 & Red-tailed Hawk (9) \\
\hline April 17 & Swainson's Hawk (1) \\
\hline March 29 & Rough-legged Hawk \\
\hline March 29 & Marsh Hawk (1) \\
\hline
\end{tabular}

Date

April 22

April 8

April 15

April 13

March 30

April 25

April 11

April 20

April 10

April 8

A pril 26

April 25

April 26

April 26

April 23

April 27

March 26

April 12

April 26

April 12

April 19

April 8

April 10

April 9

April 21

April 20

Feb. 15

April 12

April 29

March 23

April 8

March 27

April 23

April 15

April 8

April 9

April 26

April 19

March 28

April 25

March 30

April 26

April 11

April 10

April 8

April 16

April 21

March 26

April 1

March 13

April 8

April 8

April 19

Feb. 12

April 20

April 17
Spezies

Peregrine Falcon (2)

Sparrow Hawk (1)

Sandhill Crane ( 4 flocks)

American Coot (1)

Killdeer (1)

American Golden Plover (flock)

Common Snipe (2)

Willet (1)

Greater Yellowlegs

Lesser Yellowlegs

Pectoral Sandpiper

Baird's Sandpiper (19)

Marbled Godwit

Sanderling (1)

American Avocet (2)

California Gull (1)

Ring-billed Gull (1)

Franklin's Gull (1)

Bonaparte's Gull (1)

Mourning Dove (1)

Burrowing Owl (1)

Belted Kingfisher (1)

Yellow-shafted Flicker (1)

Red-shafted Flicker (1)

Eastern Phoebe (1)

Say's Phoebe (1)

Horned Lark (several)

Barn Swallow (1)

Purple Martin (1)

Common Crow (2)

Brown Creeper (3)

Robin (1)

Hermit Thrush (2)

Townsend's Solitaire (1)

Golden-crowned Kinglet (3)

Ruby-crowned Kinglet (1)

Water Pipit (several)

Myrtle Warbler (1)

Western Meadowlark (1)

Yellow-headed Blackbird (1)

Red-winged Blackbird (3)

Rusty Blackbird (2)

Brewer's Blackbird (1)

Common Grackle (1)

Purple Finch (1)

Savannah Sparrow (1)

Vesper Sparrow (1)

Slate-colored Junco (1)

Oregon Junco (1)

Tree Sparrow (1)

Fox Sparrow (1)

Song Sparrow (1)

McCown's Longspur (flocks)

Lapland Longspur (1)

Smith's Longspur (4)

Chestnut-collared Longspur (n) 